\title{
Leibniz bibliothécaire
}

\section{Stephan Waldhoff}

Traducteur : Jean-Louis Elloy

\section{(2) OpenEdition \\ Journals}

Édition électronique

URL : https://journals.openedition.org/rbnu/1612

DOI : $10.4000 /$ rbnu. 1612

ISSN : 2679-6104

\section{Éditeur}

Bibliothèque nationale et universitaire de Strasbourg

\section{Édition imprimée}

Date de publication : 1 novembre 2014

Pagination : 22-31

ISBN : 9782859230548

ISSN : 2109-2761

\section{Référence électronique}

Stephan Waldhoff, «Leibniz bibliothécaire », La Revue de la BNU [En ligne], 10 | 2014, mis en ligne le 01 novembre 2014, consulté le 17 mai 2021. URL : http://journals.openedition.org/rbnu/1612 ; DOI : https://doi.org/10.4000/rbnu.1612

\section{c) (i) (2)}

La Revue de la BNU est mise à disposition selon les termes de la Licence Creative Commons Attribution - Pas d'Utilisation Commerciale - Partage dans les Mêmes Conditions 4.0 International. 


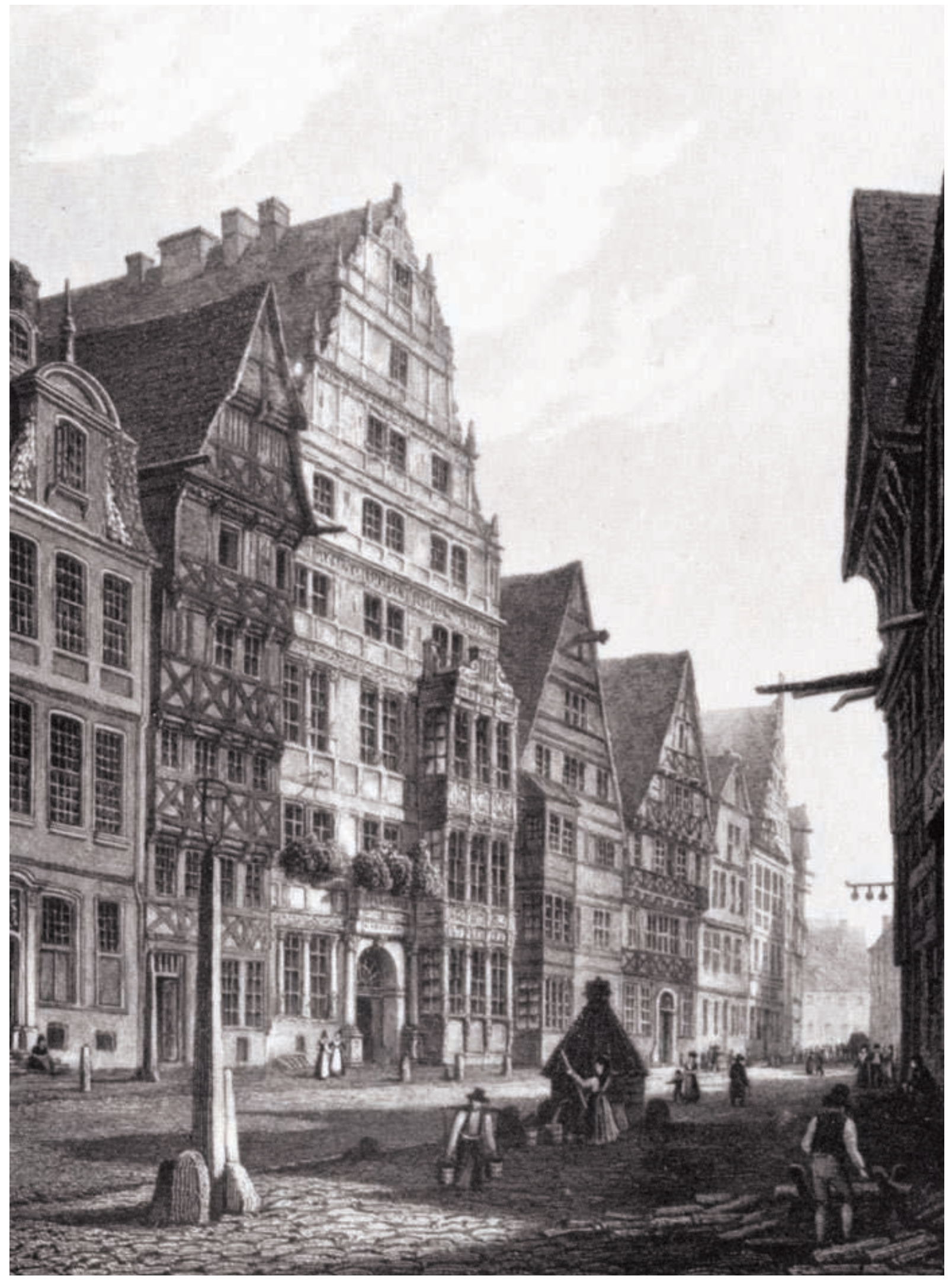

Vue du dernier lieu de résidence de Leibniz à Hanovre. Dans cette maison se trouvait aussi la bibliothèque princière (in Leben und Werk von Gottfried Wilhelm Leibniz. Eine Chronik, éd. par Kurt Müller et Gisela Krönert, Francfort/M., 1969) 


\section{Leibniz bibliothécaire}

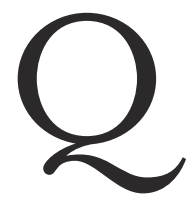

u'un érudit comme Gottfried Wilhelm Leibniz (1646-1716) ait eu affaire aux livres et aux bibliothèques, c'est l'évidence même. Tout intellectuel à son époque était nécessairement homme de livres ; chacun se constituait de riches collections, les lisait, en tirait des extraits, et pouvait échanger une correspondance ou même entreprendre un voyage dans le seul but de dénicher quelque édition rare. Rien d'étonnant donc à ce qu'un esprit aussi universel que celui de Leibniz réponde à ce profil. Mais en réalité son lien avec le monde des bibliothèques était bien plus étroit et concret que chez nombre des érudits de son temps, et ce d'un point de vue aussi bien pratique que théorique. Peu s'en faut que nous ne citions à ce propos la devise bien connue de Leibniz selon laquelle la théorie doit être liée à la pratique (" theoria cum praxi »). Mais ce serait faire appel à une formule déjà trop ressassée, et s'y référer reviendrait à se contenter d'un cliché au lieu d'expliquer le rapport contrasté et parfois disharmonieux entre les conditions pratiques d'un côté et la réflexion théorique de l'autre.

\section{Premières expériences}

Leibniz fit très tôt l'expérience des livres et des bibliothèques. Dès son jeune âge - comme il s'en souviendra plus tard - il consultait la bibliothèque érudite de son père, un professeur de Leipzig mort précocement, et c'est avec l'aide d'une édition illustrée de Tite Live qu'il s'initia lui-même aux fondements de la langue latine. Ce fils de professeur voyait s'ouvrir devant lui une voie aca- démique toute tracée pour le reste de son existence. Mais Leibniz refusa cette carrière certes sûre, mais sans grand lustre. Il ne voulait pas que ses capacités et ses connaissances fussent contraintes par l'étroitesse du monde universitaire et souhaitait plutôt les mettre au service du bien-être général.

Pour satisfaire cette ambition, il lui fallait entrer dans une cour princière. Il commença sa carrière à celle de l'archevêque de Mayence, qui joua en tant que prince électeur et archichancelier impérial un rôle déterminant dans l'organisation politique de l'Empire. Si Leibniz put, comme protestant, entrer au service du plus important prince ecclésiastique de l'Empire, c'est parce qu'un homme qui avait accompli à peu près le même parcours lui en avait frayé le chemin : Johann Christian von Boineburg (mort en 1672). C'est grâce à ce bienfaiteur que le jeune érudit fut pour la première fois confronté à la réalité pratique du travail de bibliothécaire. À la fin de sa période mayençaise (1669-1672), il se consacra en effet au catalogage de la très riche bibliothèque privée de Boineburg. Sa structuration du catalogue établit des entrées d'abord selon les langues puis selon les matières. Dans le cadre des matières, la classification s'organisait alphabétiquement en partie par mots clés, en partie par noms d'auteurs. Comme de nombreux livres pouvaient être recherchés à partir de plusieurs mots clés, Leibniz tenta de résoudre le problème en indexant les titres plusieurs fois, ou tout au moins une partie d'entre eux.

Lorsqu'il quitta Mayence, il se rendit à Paris pour y résider quatre années. Si ces années parisiennes n'ont rien apporté à son expérience de bibliothécaire, elles ont été absolument déterminantes pour son développement intellectuel. Quoiqu'il ait affirmé plus tard que 
c'était par patriotisme qu’il était revenu en Allemagne, il serait en réalité volontiers resté dans la capitale française s'il avait pu y trouver une source de revenus. De fait, il prit tout son temps pour s'installer dans ses fonctions de conseiller aulique et bibliothécaire à Hanovre, alors que ce poste lui avait déjà été proposé assez longtemps auparavant.

\section{À Hanovre}

Si après quelque hésitation Leibniz choisit de s’installer à Hanovre, où il parvint à la fin de l'année 1676 après un détour par l'Angleterre et les Pays-Bas, ce n'est pas parce qu'il aurait été particulièrement séduit par la force d'attraction de cette petite ville résidentielle de l'Allemagne du Nord. Il espérait bien plutôt, chez son nouvel employeur, le duc Johann Friedrich, entrer en relation avec un prince tel qu'il se l'était imaginé : un intellectuel d'une grande curiosité d'esprit, et donc ouvert aux idées novatrices susceptibles de contribuer au bienêtre général. Ces idées lui viendraient de son conseiller, homme de confiance et d'érudition, en l'occurrence de Leibniz lui-même. Cette fonction à laquelle il aspirait ne correspondait toutefois que très partiellement à l'emploi d'un bibliothécaire aulique. Face au duc, il s'était exprimé en termes clairs : cet emploi en tant que tel ne lui suffisait pas car " ceux qui ont commerce avec les livres sont bien peu considérés et tenus communément pour impropres à d'autres tâches" ( $\mathrm{A} \mathrm{I}, 2, \mathrm{p} .20)^{1}$. Sa charge devait, pensait-il, lui ouvrir un accès au prince et non le limiter dans son efficacité et sa sphère d'influence.

En pratique, Johann Friedrich semble avoir presque répondu à l'idéal rêvé par Leibniz. En tout cas, le duc chérissait sa bibliothèque, de dimension encore modeste au départ : dans les trois années qui suivirent l'arrivée de Leibniz, il lui consacra en moyenne plus de 1000 thalers par an (en comparaison, le financement que ses successeurs daignèrent lui accorder paraît bien ridicule). Plus significatif encore : les salles de la bibliothèque se trouvaient à proximité de ses appartements privés, et ainsi quelques enjambées séparaient Johann Friedrich de ses livres, et le bibliothécaire de son prince. Cette situation était de nature à concrétiser le rêve de Leibniz : contourner la cour et sa hiérarchie pour pouvoir, en tant que conseiller personnel du souverain, mettre en œuvre ses idées. Dans quelle mesure ce rêve a-t-il pu véritablement se réaliser ? Il est difficile de le dire. Il est tout aussi difficile d'établir, à partir des documents nombreux et bien attestés qui furent acquis pour la bibliothèque, un profil qui reflète de façon distincte les centres d'intérêt de Leibniz et ceux du prince. Quoi qu'il en soit, le nombre proportionnellement élevé d'ouvrages mathématiques ou de sciences naturelles ne passe pas inaperçu. Pour renforcer ces domaines, Leibniz avait proposé avec succès l'achat de la bibliothèque privée de l'érudit hambourgeois Martin Fogel (mort en 1675), qui comptait 3600 volumes de plus que la bibliothèque ducale au moment où Leibniz entra dans sa charge. Il semble évident que le duc a été également intéressé par ces matières, si bien que l'on peut à bon droit parler d'une convergence d'intérêt entre le prince et le bibliothécaire.

\section{Un nouvel employeur}

Mais le temps que Leibniz passa au service de Johann Friedrich fut bref. Le duc mourut à la fin de l'année 1679 et son successeur, son frère Ernst August, ne lui ressemblait guère. Il ne manifestait aucun intérêt pour les questions intellectuelles (à moins de pouvoir en tirer quelque bénéfice politique) ; son caractère ambitieux, calculateur et dépourvu de scrupules lui assura bien des succès.

$\mathrm{Au}$ siècle de Louis XIV, on se devait d'afficher son ambition politique de façon voyante. Ainsi au cours de la construction du château, une décennie à peine après le changement de souverain, on décida d'une mesure qui illustre de façon très emblématique le bouleversement de situation pour le bibliothécaire Leibniz. Pendant que ce dernier, dans le cadre de ses recherches historiques, entreprenait un voyage en Allemagne du Sud, en Autriche et en Italie (de 1688 à 169o), on fit détruire à Hanovre l'aile du château qui comportait la bibliothèque ; on voulait libérer un espace pour la construction d'un opéra. Les livres, et avec eux le bibliothécaire, furent expulsés du château et trouvèrent refuge dans une maison bourgeoise louée pour l'occasion (illustration p. 22).

Dans le château déjà, Leibniz avait eu bien du mal à maîtriser l'afflux des documents provenant de la bibliothèque de Fogel ; il parvint d'autant moins à installer correctement la bibliothèque princière dans son nouveau domicile pendant les décennies qui suivirent. Depuis qu'il avait été chargé en 1685 de rédiger l'histoire de la maison des Welf, la dynastie des princes de Hanovre, il se servait surtout de ces livres pour mener à bien ses recherches, comme s'il s'agissait d'une bibliothèque personnelle. Aussi finirent-ils par se mêler à ses propres livres. Comme il avait progressivement délaissé le travail de catalogage, il devint impossible après sa mort de distinguer quels étaient les livres du prince et 


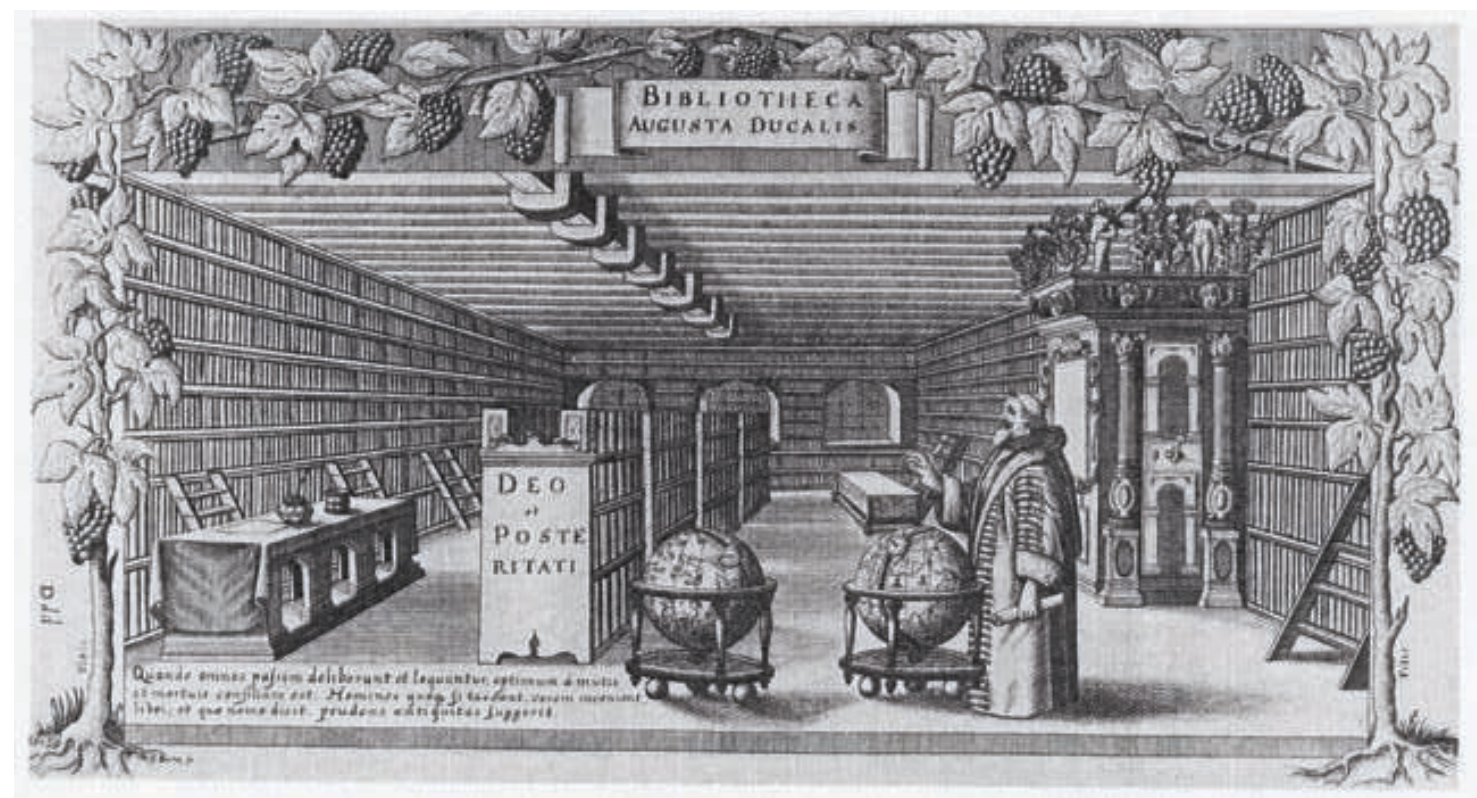

Le duc Auguste dans sa bibliothèque. On notera la grande précision de la représentation, dont les détails sont confirmés par une description réalisée par Leibniz (in Martin Gosky, Arbustum vel Arboretum Augustæum, 1650)

quels étaient les siens. Aujourd'hui encore l'incertitude demeure car le problème fut résolu alors de la façon suivante : le souverain dédommagea les héritiers et devint propriétaire de toute la succession, dont la bibliothèque privée de Leibniz. De son vivant encore, ce dernier avait fini par interdire aux visiteurs l'accès aux fonds en raison de leur état de désordre. Ce fait révèle clairement qu'il ne se souciait plus guère de se montrer exemplaire dans la gestion de la bibliothèque.

Mais nous avons un peu trop anticipé. Revenons-en une fois encore à l'année 1680, l'année du changement de souverain. Le poste de Leibniz était alors remis en question, comme tous les autres emplois équivalents. Quoiqu'il ait été prévenu assez tôt qu'il y serait maintenu, il réagit rapidement face à cette nouvelle situation. Ce n'est pas un hasard s'il rédigea en cette même année toute une série de mémoires sur l'administration de l'État, dont un sur l'organisation d'une bibliothèque. Celui-ci commence en ces termes : " Une Bibliotheque doit estre un inventaire general, un soulagement de la memoire, un Archif imprimé " (A IV, 3, p. 350). Cette dernière expression que Leibniz a répétée à de nombreuses reprises ne doit pas être comprise à tort. Le terme d'" archif " ne signifie pas ici le lieu dépositaire d'un savoir historiographique érudit, mais désigne bien plutôt un ensemble d'instruments grâce auxquels on se prépare à un exercice légitime du pouvoir politique. C'est pourquoi Leibniz centre sa réflexion sur des sujets comme les entreprises de fortification, l'économie et les manufactures, les cérémonies, les fêtes et l'architecture, sujets tous en rapport étroit avec le pouvoir princier et le déploiement de ses fastes.

Il va sans dire que ce discours va dans le sens des intérêts de son nouveau maître et employeur. Il serait faux cependant de n'y voir qu'une manifestation d'opportunisme. Une réflexion sur le statut des bibliothèques s'y dessine partiellement et il ne conviendrait pas de la négliger. Leibniz voulait que la science, tout comme les instruments dont elle avait besoin, serve la politique et le bien commun. Il fallait, à ses yeux, que la politique fût bien informée pour pouvoir être rationnelle et génératrice de prospérité pour les sujets du souverain. Une bibliothèque bien pourvue, comme le montrent les expressions qui précèdent, ne constituait qu'un des rouages participant au rassemblement des informations, à leur ordonnancement et à leur enregistrement. De véritables archives et un cabinet de curiosités devaient seconder l' " Archif imprimé ". 
Le trésor de connaissances, constitué par la convergence des informations provenant du pays tout entier, devait ensuite être à nouveau diffusé à travers ce même pays afin que le progrès gagnât tous les niveaux du savoir.

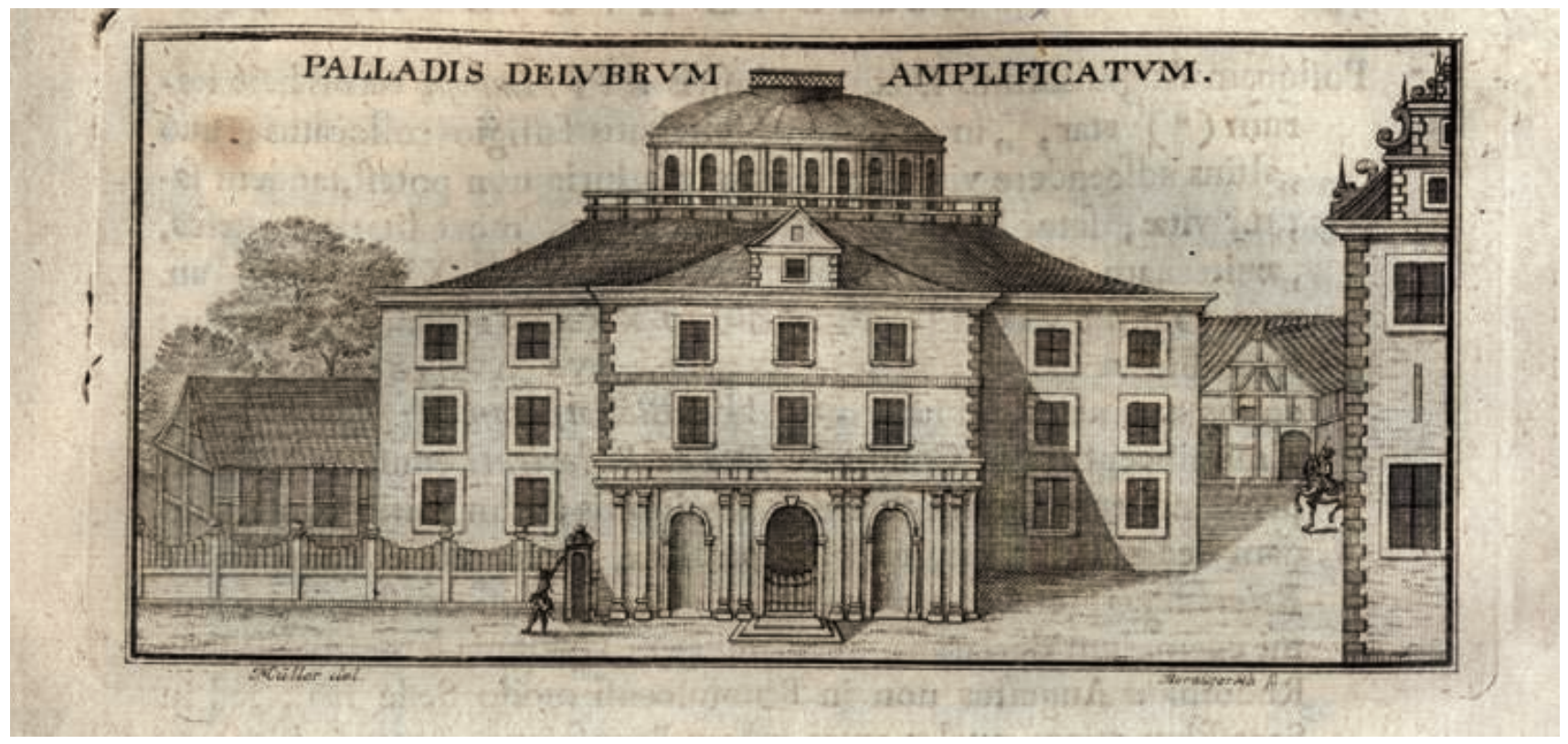

La « rotonde " a été construite de 1706 à 1710 ; c'est l'un des premiers bâtiments,

au nord des Alpes, spécifiquement construits pour abriter une bibliothèque

(in Jacob Burckhard, Historia bibliothecae Augustae [pars 1], Wolfenbüttel, s. d.) 
Leibniz se montre ainsi moins novateur dans sa conception de la bibliothèque que dans celle des archives et du cabinet de curiosités. Ces derniers avaient pour vocation d'accueillir et de rassembler les informations et les données de tout un territoire, et ce non seulement pour des faits pouvant intéresser les juristes ou des données statistiques, mais aussi pour ce qui concernait les techniques artisanales anciennes ou les nouvelles technologies. Sur ce point, Leibniz allait au-delà de la science traditionnelle du livre et annonçait déjà d'une certaine façon les objectifs de l'Encyclopédie. Le trésor de connaissances, constitué par la convergence des informations provenant du pays tout entier, devait ensuite être à nouveau diffusé à travers ce même pays afin que le progrès gagnât tous les niveaux du savoir. C'était le point central d'un grand nombre de ses projets de réforme.

Mais il ne s'en tint pas là : il ne s'intéressa pas qu'à la constitution de collections, mais aussi à l'organisation et à la concentration des informations. Les archives devaient, comme une pyramide, être coiffées d'une pointe, d'un ouvrage de référence, qu'il désigne par l'expression de " tables de l'État ", et qui aurait été destiné à offrir au prince un aperçu rapide sur les informations essentielles (A IV , 3, $n^{\circ} 29$ ). On retrouve une préoccupation semblable dans le domaine du livre et de l'édition quelque temps auparavant, avec ses considérations pour l'établissement d'un répertoire semestriel des publications essentielles (Nucleus librarius semestralis; A I, 1, $\mathrm{n}^{\circ}$ 2), une sorte de catalogue périodique destiné à paraître avant chaque foire de Francfort, le rendez-vous le plus important pour le commerce du livre. Par de telles innovations dans l'organisation et la transmission synthétique du savoir, Leibniz tentait de contenir le flot des informations, qu'à son époque déjà un esprit universel comme le sien ne pouvait plus embrasser d'un seul regard.

\section{Wolfenbüttel}

À sa charge de bibliothécaire à Hanovre s'ajouta en 1691 celle de Wolfenbüttel, où résidait une autre branche de la maison des Welf. La petite ville résidentielle hébergeait l'une des plus importantes bibliothèques d'Europe, la Bibliotheca Augusta, ainsi nommée en mémoire de son fondateur, le duc Auguste le Jeune (mort en 1666 ; illustration p. 25). Leibniz en fit l'égale de la Bibliothèque impériale de Vienne ou de la Bibliothèque royale de Paris. S'il obtint l'autorisation d'avoir cette charge, cela tient certainement au fait qu'il n'était pas contraint d'être constamment présent à Wolfenbüttel, mais aussi à la tâche qu'on lui avait confiée depuis 1685, la rédaction de l'histoire dynastique. Comme bibliothécaire, il pouvait plus efficacement exploiter ces riches trésors livresques dans le cadre de ses études historiques.

Certes, à Wolfenbüttel également, Leibniz dut essuyer quelques déceptions. En se préparant à son emploi, il avait imaginé d'ambitieux projets pour le développement futur de la bibliothèque. Ces derniers n'étaient possibles qu'à condition de disposer d'un budget régulier d'acquisition, dont Leibniz avait fixé le montant à 1 ooo thalers. Cette condition s'avéra bien vite illusoire. Son budget d'achat se limita à 100 thalers qui furent d'ailleurs versés de manière fort irrégulière. Leibniz pouvait craindre que la postérité associât le déclin de la Bibliotheca Augusta, autrefois si glorieuse, à la période de son mandat de bibliothécaire. Il ne faudrait pas s'imaginer cependant que face à ces moyens modestes il se soit contenté de gémir sur son sort. Bien au contraire : le manque de ressources financières l'incita à découvrir d'autres sources de revenus pour la bibliothèque. Il proposa ainsi l'introduction de papier estampillé (une forme de taxe administrative) puis quand cette idée fut refusée, il suggéra de planter des mûriers destinés à la sériculture. En tout cas, quand il eut pris la mesure du caractère illusoire de ses ambitieux projets initiaux, il se recentra sur des tâches nécessaires et concrètement réalisables... non sans succès : on lui doit le catalogue alphabétique des auteurs qui aujourd'hui encore sert de fondement au catalogue informatique.

Pendant la période où Leibniz exerça son emploi, on édifia à Wolfenbüttel l'un des premiers bâtiments au nord des Alpes exclusivement réservés à la fonction de bibliothèque. Mais la célèbre rotonde (illustration p. 26), un bâtiment central couronné d'un globe céleste, avait été construite en colombage, et elle se délabra rapidement, au point qu'elle dut être détruite au $19^{\mathrm{e}}$ siècle. La tentation est grande d'établir un lien entre cette conception architecturale et le savant universel qu'était Leibniz, de retrouver la représentation de ses idéaux dans les plans de construction. Leibniz a-t-il eu une influence dans l'érection de ce nouveau bâtiment ? Si oui, dans quelle mesure ? La question est controversée. Il n'existe pas de preuves avérées selon lesquelles il aurait participé à l'établissement des plans.

Ce qui est certain, c'est qu'il n'a pas été impliqué dans la commande visant à acheter une bonne centaine de manuscrits, presque tous médiévaux, en provenance du monastère alsacien de Wissembourg. C'est son prédécesseur qui en 1689 l'avait engagée. Cela n'empêcha pas Leibniz de réclamer pour la bibliothèque l'un des 
" codices " de ce fonds, qui avait fait l'objet d'un prêt de la part de son propriétaire ; et il parvint à l'obtenir. Il établit par ailleurs des notices pour quelques manuscrits provenant de Wissembourg, ce qui témoigne aussi de son vif intérêt pour ces derniers.

Le fonds précieux des manuscrits de Wolfenbüttel ne l'aida pas seulement dans ses recherches historiques : il y trouva matière à publications pour les plus grands profit et rayonnement de la bibliothèque (l'un de ses plus célèbres successeurs, Gotthold Ephraim Lessing, bibliothécaire à Wolfenbüttel à partir de 1770, le suivit dans cette voie). La moitié des pièces au moins de son Codex juris gentium diplomaticus provient de cette source (illustration p. 29). Aussi a-t-il spécifié à bon droit sur la page de titre : "A partir avant tout des manuscrits de la Bibliotheca Augusta de Wolfenbüttel ». Pour la rédaction de ce Codex, il s'appuya surtout sur le fonds des manuscrits dits "mazarins". Il s'agit de presque 400 volumes de textes reproduisant des papiers du cardinal Mazarin et d'autres hommes d'État français ; le duc Auguste avait obtenu l'autorisation d'établir ces copies à Paris, pour la somme énorme de 24 ooo thalers. D'un point de vue moderne, ces sources restent douteuses, mais pour Leibniz elles étaient riches en contenus scientifique et politique. Sous ce double rapport, il sut tirer profit de cette abondante documentation (en principe très confidentielle) sur les puissances gouvernantes.

\section{La bibliothèque comme « cosmos du savoir "}

Si l'activité de Leibniz à Wolfenbüttel consista avant tout en une gestion pratique de la bibliothèque et s'il utilisa les fonds pour approfondir ses connaissances historico-juridiques, l'abondance des livres qui s'y trouvaient l'inspira cependant pour d'autres projets. Toute une série d'essais pour l'organisation systématique d'une bibliothèque universelle (A IV, $5, \mathrm{n}^{\text {os }} 70-80$; A IV, $\left.6, \mathrm{n}^{\text {os }} 65-70,72,77-78\right)$ datent pour l'essentiel des années 169o, c'est-à-dire suivent sa prise de fonction en tant que bibliothécaire à Wolfenbüttel. Ces essais furent d'ailleurs souvent rédigés sur le papier que Leibniz utilisait là-bas.

Deux d'entre eux, consacrés à la conception d'un classement systématique des bibliothèques, furent publiés dès 1718 ; de façon décisive et durable, ils donnèrent de Leibniz l'image d'un théoricien de la bibliothèque - et dans le même temps gauchirent la réalité. Leur premier éditeur les définit comme présentant "l'idée leibnizienne d'une bibliothèque publique structurée selon les ca- tégories du savoir " et vit dans ces deux essais une version longue, plus détaillée (A IV, $5, \mathrm{n}^{\circ} 81$ ), et une version courte, abrégée (A IV, $6, \mathrm{n}^{\circ}$ 72), d'un même projet et système. Cette façon de voir ne fut pas remise en question par la suite, quoique l'essai le plus court ne puisse en aucune façon être compris comme un simple résumé de l'essai le plus long, et qu'il s'en écarte au contraire sur bien des points. Que l'on mette ces deux textes en perspective avec les autres essais : ils apparaissent alors comme deux instantanés fixant deux phases de cette longue lutte menée par Leibniz pour l'élaboration d'un classement des bibliothèques, deux instantanés qui se rapportent d'ailleurs à des moments bien distincts de cette élaboration.

Trompée par cette interprétation, la postérité a souvent été amenée à établir un lien trop étroit entre les essais de Leibniz et la pensée du fameux théoricien des bibliothèques Gabriel Naudé ${ }^{2}$. Certes Leibniz s'en est parfois inspiré. On peut même, dans leur conception respective du classement systématique, trouver d'importantes convergences, à première vue en tout cas. Naudé avait précisé : " ie croy que le meuilleur [ordre] est tousjours celuy qui est le plus facile, le moins intrigué, le plus naturel, vsité, \& qui suit les Facultez de Theologie, Medecine, Iurisprudence, Histoire, Philosophie, Mathematiques, Humanitez, \& autres [...] " ${ }^{3}$. Leibniz lui aussi s'est référé au classement traditionnel des facultés universitaires. Ce qui différencie sa conception de celle de Naudé tient aux deux mots " et autres " en fin de citation. Jamais il n'aurait pu les faire figurer ainsi, comme clausule, dans l'énoncé de son projet. S'il avait seulement importé à Leibniz, comme à Naudé, de construire un classement simple, communément connu et reconnu, il n'aurait sans doute rien trouvé à y redire ; mais son ambition allait au-delà. Ses essais tentent d'édifier le " cosmos du savoir " ${ }^{4}$ au plein sens du terme : un ordonnancement autonome et pleinement signifiant.

Deux tentatives témoignent de ces efforts. Pour commencer, il prend soin de classer et de désigner les territoires du savoir qui échappaient aux trois facultés supérieures, la théologie, la jurisprudence et la médecine. Or c'est dans ces domaines, pendant les temps modernes, que les connaissances se développèrent le plus, sur un rythme toujours plus rapide. Il devenait de plus en plus difficile de placer sous la tutelle de la faculté de philosophie ces nouvelles branches du savoir qui aspiraient à l'indépendance. Au $19^{\mathbf{e}}$ siècle, elles feront éclater, surtout les sciences de la nature, la classification traditionnelle de l'université. C'est précisément dans les sciences que 


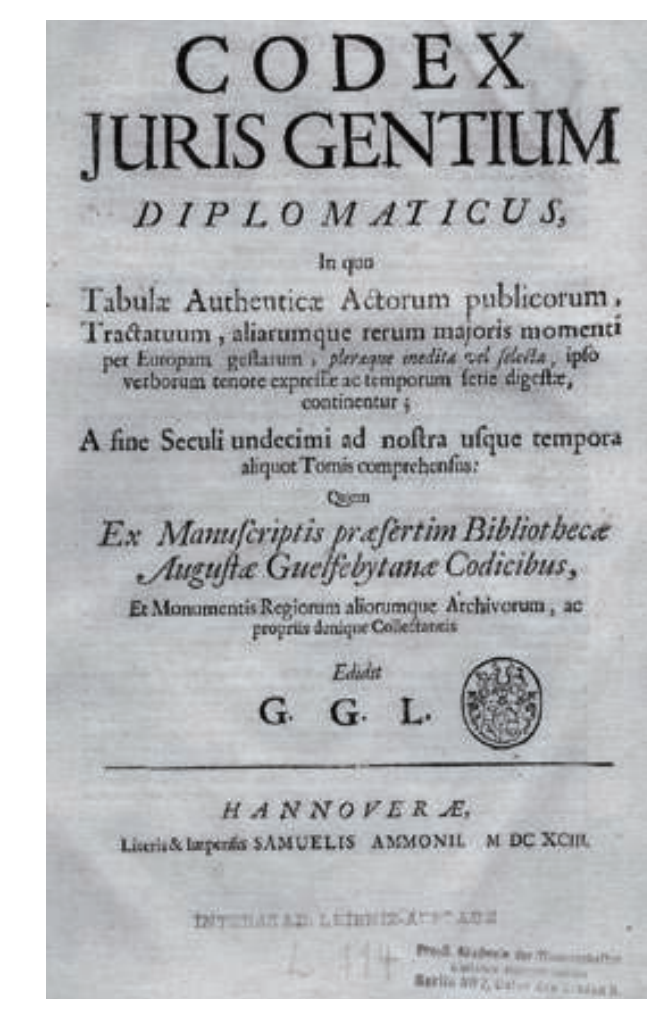

Page de titre du Codex juris gentium diplomaticus de 1693 (coll. Berlin - Brandenburgische Akademie der Wissenschaften)

les considérations de Leibniz sur les bibliothèques et leur classement s'avèrent les plus intéressantes ; on peut y suivre l'évolution de sa pensée, continuellement en quête de terminologies nouvelles et d'autres configurations disciplinaires.

A l'intérieur de chaque discipline, Leibniz affine toujours plus la distinction des subdivisions catégorielles, en définissant les différentes branches et sous-branches d'un même savoir. Par ailleurs, dans l'un des essais (illustration p. 30), on retrouve une des préoccupations que nous avons eu déjà l'occasion de mentionner précédemment : offrir le tableau synthétique non bien sûr du savoir tout entier, mais de son ordonnancement général, de manière à ce qu'il puisse être embrassé et compris d'un seul regard.

De telles représentations finissent par s'éloigner sensiblement du domaine des livres. Ainsi dans ces essais presque aucun titre d'ouvrage n'est cité. Ce n'est pas très étonnant : si l'on suit ces réflexions jusque dans leurs ultimes et leurs plus subtiles ramifications, on ne peut s'empêcher de penser que Leibniz a quelque peu perdu de vue son but initial, la classification systématique des livres, et qu'il a fini par décrire le " cosmos du savoir" dans une perspective purement abstraite.
Parallèlement à ces essais d'une grande sophistication, Leibniz rédigea à plusieurs reprises des répertoires bibliographiques, structurés de manière bien plus simple et qui, eux, contiennent dans chacune de leurs rubriques de véritables listes de livres. Le premier exemple se rencontre assez tôt, dans l'opuscule Nova methodus discendae docendaeque jurisprudentiae de 1667 (A VI, 1, $\mathrm{n}^{\circ} 10$ ). Cet écrit, qui envisage une réorganisation de l'étude des sciences juridiques, propose également une liste de lectures ordonnées thématiquement, centrée bien évidemment sur le domaine du droit mais qui, dans sa thématique, va bien au-delà de ce que l'on pourrait exiger d'un étudiant d'aujourd'hui. Deux décennies plus tard, il catalogua une liste de titres pour un homme d'État viennois qui cherchait à se constituer une bibliothèque privée à vocation universelle (A I, 5, $\left.\mathrm{n}^{\circ} 247\right)$. Et dans la dernière année de son existence, vraisemblablement peu de temps avant sa mort, il commença un projet similaire mais dont nous ne connaissons ni les circonstances, ni le destinataire. Une main étrangère a porté sur ce texte l'indication suivante : "Recommandations de G. W. Leibniz pour la classification d'une bibliothèque ". Il en existe un brouillon autographe et une copie faite par un secrétaire. Ces deux documents s'interrompent soudainement. La mort a-t-elle surpris le bibliothécaire Leibniz en pleine rédaction? 


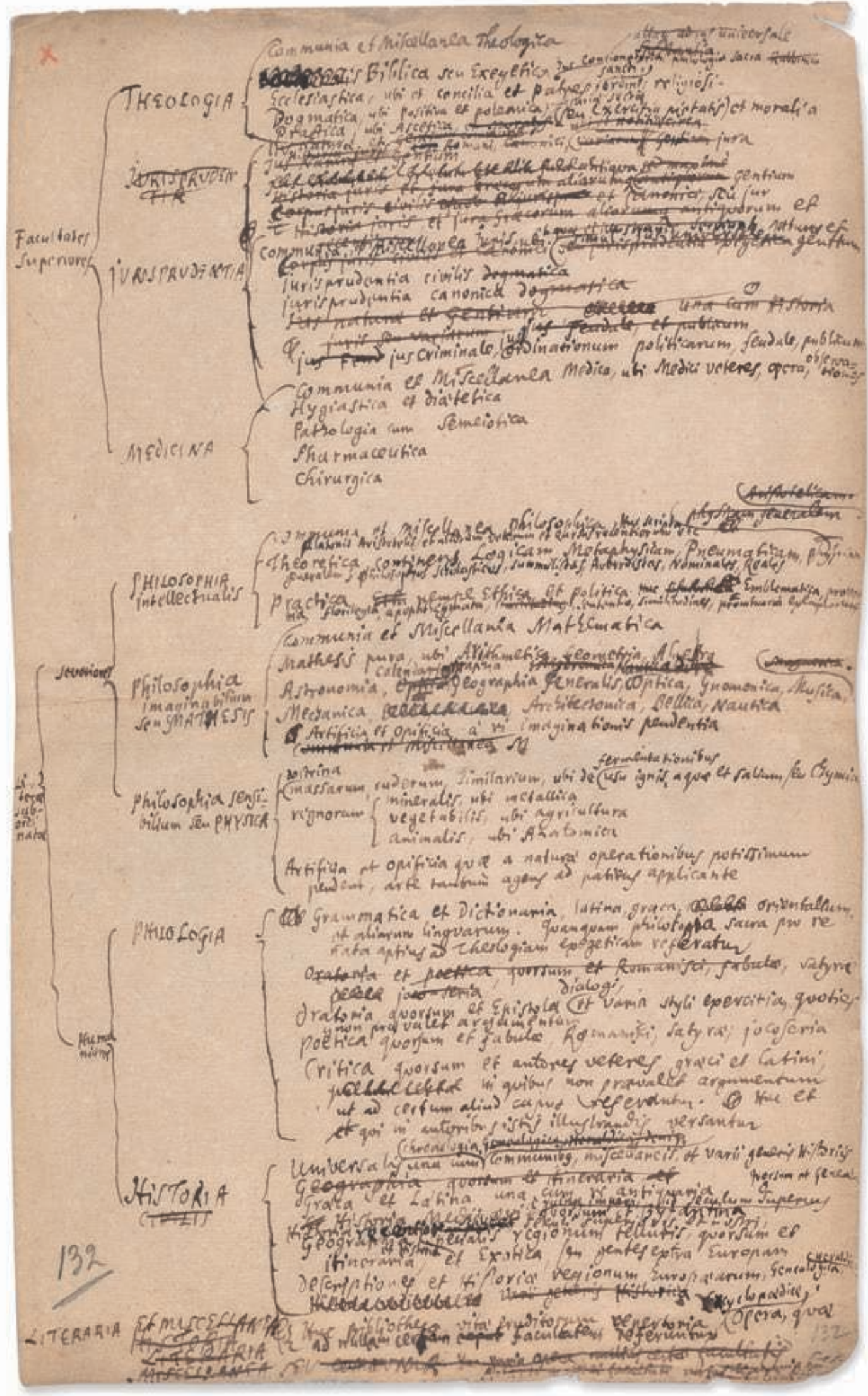

Cette esquisse d'un classement systématique de bibliothèque, sous la forme d'un tableau et de sections (A IV, $6, \mathrm{n}^{\circ} 77$ ) permettait d'englober pour ainsi dire en un regard le « cosmos du savoir » (coll. Niedersächsische Landesbibliothek). 


\section{En guise de conclusion}

Le Leibniz bibliothécaire et théoricien des bibliothèques est toujours resté présent dans les mémoires. Mais le fait qu'il ait consigné ses réflexions dans des lettres, des mémoires et des essais, sans systématiquement les rassembler ni les publier, a tout d'abord empêché leur réception auprès de ses contemporains et de la postérité. La science des bibliothèques a toutefois plus que compensé cette lacune. Eu égard à son influence réelle, Leibniz y occupe une place historique excessivement importante. Qui plus est, l'insuffisance des sources textuelles a pendant longtemps faussé les perspectives. Tantôt ses réflexions ont été considérées comme simplement tributaires des théoriciens qui l'avaient précédé ; tantôt - et cela se justifie encore moins - on a exagéré sa modernité, on en a même fait un précurseur de la classification décimale de Melvil Dewey ${ }^{5}$. En réalité, l'apport significatif de Leibniz en tant que théoricien des bibliothèques tient peu à l'originalité présumée de ses positions - presque toutes avaient déjà été imaginées et formulées çà et là. Il se distingue bien plutôt pour s'être saisi de la notion de " cosmos du savoir ", emblématique de son époque, afin de bâtir une classification systématique des bibliothèques. Mais le plus remarquable demeure l'exceptionnelle pertinence de vue dont il fait preuve pour concevoir une institution qui, associant la bibliothèque dépositaire du savoir avec les archives et les cabinets de curiosités, devait permettre à l'État d'assurer une administration rationnelle et éclairée, au service de l'amélioration du genre humain. Il posait par là les fondations d'une "société de la connaissance ", pour reprendre une notion bien en vogue aujourd'hui, et que nous croyons être d'invention récente.

\section{Stephan Waldhoff \\ (traduction Jean-Louis Elloy)}

\section{Notes}

\author{
1 - Ce renvoi, ainsi que ceux qui suivent, se rapporte à l'édition de référence, \\ encore en cours de publication : Gottfried Wilhelm Leibniz, Sämtliche \\ Schriften und Briefe, Darmstadt, 1923- , Berlin, 1950-. Le chiffre romain \\ désigne la série, le chiffre arabe le volume; ils sont suivis d'un numéro \\ d'exemplaire ou d'une indication de page. Les réflexions de Leibniz sur \\ les bibliothèques se retrouvent disséminées dans des lettres et dans \\ des écrits divers (voir la note suivante). Les textes traitant de ce sujet \\ appartiennent surtout aux séries I (Allgemeiner, politischer und historischer \\ Briefwechsel) et IV (Politische Schriften). \\ 2 - L'éditeur de l'ouvrage de Naudé, Advis pour dresser une bibliothèque \\ (paru pour la première fois à Paris en 1627), signale dans l'édition de \\ Leipzig publiée en 1963 (p. 125) que les textes de Leibniz l'avaient tout \\ d'abord intéressé. Mais comme " ses pensées relatives à une réforme \\ du statut des bibliothèques allemandes se dispersaient au gré des \\ contributions, considérations et lettres diverses ", il n'avait pas jugé bon \\ de les éditer, préférant jeter son dévolu sur « un autre classique plus \\ ancien parmi les théoriciens des bibliothèques - sur Gabriel Naudé. \\ De toute évidence l'œuvre de Leibniz lui était redevable sur de nombreux \\ points $[\ldots]$ ". \\ 3 - Ibid., chap. 7, p. 100 \\ 4 - Uwe Jochum, Kleine Bibliotheksgeschichte, Stuttgart, $2^{e}$ éd., 1999, p. 187 \\ 5 - Heinrich Lackmann, Leibniz' bibliothekarische Tätigkeit in Hannover, \\ in Leibniz, sein Leben - sein Wirken - seine Welt, éd. par Wilhelm Totok \\ et Carl Hasse, Hannover, 1966, p. 321-348, ici p. 335
}

\section{ORIENTATIONS BIBLIOGRAPHIQUES :}

Pour ce qui concerne la bibliographie critique sur Leibniz, on trouvera les références essentielles dans les ouvrages suivants : Leibniz-Bibliographie. Die Literatur über Leibniz bis 1980, Frankfurt/M., $2^{\mathrm{e}}$ éd., 1984, p. 99-103, et Leibniz-Bibliographie. Band 2: Die Literatur über Leibniz 1981-199o, Frankfurt/M., 1996, p. 23-24.

Pour les années suivantes, on pourra trouver des compléments d'information sur le site : www.leibniz-bibliographie.de

On ne mentionnera ici que quelque parutions récentes :

- Karin Hartbecke (éd.), Zwischen Fürstenwillkür und Menschheitswohl - Gottfried Wilhelm Leibniz als Bibliothekar, Frankfurt/M., 2008

- À propos de l'activité de Leibniz à Wolfenbüttel, qui n'est pas traitée dans le précédent ouvrage : Günter Scheel, Leibniz' Beziehungen zur Bibliotheca Augusta in Wolfenbüttel (1678-1716), in Braunschweigisches Jahrbuch, 54, 1973, p. 172-199.

- Sur ses positions par rapport à la librairie et sur des sujets analogues : Annegret Stein-Karbach, Leibniz und der Buchhandel, in Archiv für Geschichte des Buchwesens, 23, 1982, p. 1189-1418 (disponible également dans une édition séparée parue en 1983). 